\title{
The Incompressible Limit in Nonlinear Elasticity
}

\author{
Steven Schochet* \\ Department of Mathematics, Princeton University, Princeton, NJ 08544, USA
}

Abstract. The incompressible limit in nonlinear elasticity is shown to fall under the theory of singular limits of quasilinear symmetric hyperbolic systems developed by Klainerman and Majda. Specifically, initial-value problems for a family of hyperelastic materials with stored energy functions

$$
W\left(\frac{\partial x}{\partial X}\right)=W_{\infty}\left(\frac{\partial x}{\partial X}\right)+\lambda^{2} w\left(\operatorname{det} \frac{\partial x}{\partial X}\right)
$$

are considered, where $X$ and $x$ are reference and deformed coordinates respectively. Under the assumption that the elasticity tensor

$$
A_{k l}^{i j} \equiv \frac{\partial^{2} W_{\infty}}{\partial\left(\frac{\partial x^{i}}{\partial X^{k}}\right) \partial\left(\frac{\partial x^{j}}{\partial X^{l}}\right)}
$$

is positive definite near the identity matrix and that $w^{\prime \prime}(1)>0$, the following results are proven for appropriate initial data:

i) existence of solutions of the corresponding evolution equations on a time interval independent of $\lambda$ as $\lambda \rightarrow \infty$, and ii) convergence as $\lambda \rightarrow \infty$ of the solutions to a solution of the incompressible elastodynamics equations.

\section{Introduction}

The incompressible limit in fluid dynamics has received considerable attention in the last few years $[2,6,7,11,15]$. The basic result, which has been proven in various contexts, is that slightly compressible fluid flow is close to incompressible flow, even though the equations for the latter are related to those for the former via a singular limit. This justifies the use of the incompressible flow equations for certain real fluids that are actually slightly compressible.

A similar situation occurs in elasticity: certain types of rubber strongly resist changes in volume, and so are often modelled as incompressible solids. This procedure has been justified for elastostatics by Rostamian [10]; our goal here is to justify it, at least in certain contexts, for hyperelastic dynamics by applying the

* Supported by NSF postdoctoral fellowship \#DMS-84-14107 
theory of singular limits of quasilinear symmetric hyperbolic systems developed by Klainerman and Majda [6,7] for the fluid dynamics case.

The elastodynamic equations for a compressible hyperelastic material are (see $[3,13])$

$$
D_{t}^{2} x^{i}=D_{k} \frac{\partial W}{\partial F_{k}^{i}},
$$

where $x(t, X)$ is the position of time $t$ of the particle whose reference position is $X$,

$$
\left.D_{t} \equiv \frac{\partial}{\partial t}\right|_{X \text { fixed }},\left.\quad D_{k} \equiv \frac{\partial}{\partial X^{k}}\right|_{t \text { fixed }}, \quad F_{k}^{i} \equiv \frac{\partial x^{i}}{\partial X^{k}},
$$

and $W(F)$ is stored energy function of the material. For convenience it has been assumed that the density is identically one in the reference state and that there are no body forces. We will be considering initial-value problems for a family of hyperelastic materials in $R^{n}$ characterized by stored energy functions

$$
W_{\lambda}(F)=W_{\infty}(F)+\lambda^{2} w(J)
$$

where

$$
J \equiv \operatorname{det} F=\operatorname{det} \frac{\partial x^{i}}{\partial X^{k}},
$$

and where $w$ will be assumed to satisfy

$$
w^{\prime \prime}(1)>0 .
$$

Since a term affine-linear in $J$ can be added to $W_{\lambda}$ without affecting the dynamics (see (2.4-2.5) below), one might as well assume also that $w(1)=0=w^{\prime}(1)$, so that in a formal sense $\lambda=\infty$ clearly imposes the incompressibility constraint $J=1$.

$W_{\infty}$ could be allowed to depend also on $(t, X, x)$ without changing the results to be presented.

Previous proofs of existence for the initial-value problem of elastodynamics $([4,5])$, when applied to hyperelastic materials with stored energy functions $W_{\lambda}$, yield solutions for times $T(\lambda)$ that might go to zero as $\lambda \rightarrow \infty$. We will show, under conditions specified later, that the solutions actually exist on a fixed time interval, $[0, T]$, and converge, as $\lambda \rightarrow \infty$, to a solution of the elastodynamic equations for an incompressible material with stored energy function $W_{\infty}$. Thus, slightly compressible elastodynamics is close to incompressible elastodynamics. Note also that in particular this proves existence of solutions to the initial-value problem for incompressible elasticity.

These results are obtained by converting the elasticity equations (1.1) to a first order system and noticing that this system satisfies the structural conditions from $[6,7]$.

In order to do this, it is necessary to assume that the elasticity tensor $A_{k l}^{i j}$, defined by

$$
A_{k l}^{i j}(F)=\frac{\partial^{2} W_{\infty}}{\partial F_{k}^{i} \partial F_{l}^{j}}
$$


is positive definite in a neighborhood of the identity, i.e. that

$$
A_{k l}^{i j}(F) z^{i k} z^{j l} \geqq C|z|^{2},
$$

for all $z \in R^{n^{2}}$ and all $F$ in a neighborhood of the identity matrix. For general symmetric $A_{k l}^{i j}$, assumption (1.6) is stronger than the more commonly assumed Legendre-Hadamard condition

$$
A_{k l}^{i j}(F) z^{i} z^{j} y^{k} y^{l} \geqq C|z|^{2}|y|^{2} .
$$

(See [1 and 14] and the references there.) However, given a stored-energy function $W$ for which (1.7) holds, it often happens that a $W_{N L}$ can be found such that (i) the equations of motion do not change when $W$ in (1.1) is replaced by $W+W_{N L}$ (i.e. $W_{N L}$ is a null-Lagrangian), and (ii) the $A_{k l}^{i j}$ for $W+W_{N L}$ satisfy (1.6). For a planar material this is always true $([9,12])$. In three dimensions it has been shown true when $A_{k l}^{i j}$ at the identity has the classical form

$$
\left[\left(C_{1}\right)^{2}-2\left(C_{2}\right)^{2}\right] \delta_{i k} \delta_{j l}+\left(C_{2}\right)^{2}\left(\delta_{i j} \delta_{k l}+\delta_{k j} \delta_{i l}\right),
$$

where $C_{1}>C_{2}>0$, with $W_{N L}$ being the sum of $2 \times 2$ subdeterminants $\sum_{r<s} F_{r}^{r} F_{s}^{s}-F_{s}^{r} F_{r}^{s}([5])$. Assumption (1.6) (or (1.7)) is what distinguishes elasticity from fluid dynamics, for which, as will become evident later, $W_{\lambda}=\lambda^{2} w(J)$; i.e. the $A_{k l}^{i j}$, as defined here, vanish identically.

System (1.1) will be expressed more explicitly as a second order system, and then converted to a first order one, in Sect. 2; the uniform existence and convergence theorems will be proven in Sect. 3 .

\section{Coordinate Systems and Equations of Motion}

Let $X$ denote the reference coordinate system of $R^{n}$, and $x(t, X)$ the position at time $t$ of the particle with reference position $X$. The function $x(0, X)$ will be $C^{1}$ and invertible, and the equations of motion will guarantee that the same is true for $x(t, X)$ on some interval $[0, T]$. Its inverse $X=X(t, x)$ is also $C^{1}$ by the inverse function theorem, and $\partial X / \partial x$ is the inverse of $\partial x / \partial X$. It will be convenient to define

$$
u=x-X, \quad G=\left(G_{k}^{i}\right)=\left(F_{k}^{i}\right)-I=\frac{\partial x^{i}}{\partial X^{k}}-\delta_{k}^{i},
$$

since $u$, and not $x$, will be square integrable.

The Lagrangian derivative operators defined in (1.2) and the Eulerian ones defined by

are related by

$$
\partial_{i}=\left.\frac{\partial}{\partial x^{i}}\right|_{t \text { fixed }}, \quad \partial_{t}=\left.\frac{\partial}{\partial t}\right|_{x \text { fixed }}
$$

$$
\partial_{i}=\frac{\partial X^{k}}{\partial x^{i}} D_{k}, \quad \partial_{t}=D_{t}-v^{i} \partial_{i}
$$


where

$$
v \equiv D_{t} x
$$

Plugging $W=W_{\lambda}$ defined by (1.3) into the elastodynamic equations (1.1) and writing the result in terms of $u$ and $G$ gives

$$
D_{t}^{2} u^{i}=D_{k}\left(\frac{\partial W_{\infty}(I+G)}{\partial G_{k}^{i}}\right)+\lambda^{2} D_{k}\left(\frac{\partial}{\partial G_{k}^{i}} W(J)\right)
$$

The first term on the right of (2.2) can be written as

$$
D_{k}\left(\frac{\partial W_{\infty}(I+G)}{\partial G_{k}^{i}}\right)=A_{k l}^{i j}(I+G) D_{k} D_{l} u^{j}
$$

where the $A_{k l}^{i j}$ are defined in (1.5). In order to simplify the second term in the right of (2.2), it is useful to have the formulas

$$
\frac{\partial J}{\partial G_{k}^{i}}=\frac{\partial J}{\partial F_{k}^{i}}=J \frac{\partial X^{k}}{\partial x^{i}}
$$

and

$$
D_{k} J\left(\frac{\partial X^{k}}{\partial x^{i}}\right)=0
$$

the first of which is just Cramer's rule and the second of which is an easy exercise in linear algebra. It is also convenient to define

$$
\rho \equiv J^{-1}, \quad P(\rho) \equiv-w^{\prime}(1 / \rho)
$$

The quantity $\rho$, which represents the density of the deformed material, satisfies the conservation-of-mass equation

$$
D_{t} \rho+\rho \partial_{i} v^{i}=0
$$

as can be seen by calculating $D_{t} J^{-1}$ using the chain rule and (2.4). Since given $x(t, X), \rho=J^{-1}$ is the unique solution of (2.7) with initial data

$$
\rho(0, X)=1 / \operatorname{det}\left(\frac{\partial x(0, X)}{\partial X}\right)
$$

$\rho$ can be viewed as being defined by (2.7-2.8) rather than (2.6). Similarly, the incompressibility constraint $J \equiv 1$ can equally well be imposed by the condition $\partial_{i} v^{i} \equiv 0$ and initial condition $J(t=0) \equiv 1$.

Using (2.4-2.5), the second term on the right of (2.2) can be written as

$$
D_{k}\left(\frac{\partial}{\partial G_{k}^{i}} w(J)\right)=D_{k}\left(w^{\prime}(J) \frac{\partial J}{\partial G_{k}^{i}}\right)=D_{k}\left(w^{\prime}(J) J \frac{\partial X^{k}}{\partial x^{i}}\right)=J \frac{\partial X^{k}}{\partial x^{i}} D_{k} w^{\prime}(J)
$$

which in turn can be expressed either as

$$
J \frac{\partial X^{k}}{\partial x^{i}} D_{k} w^{\prime}(J)=J^{2} w^{\prime \prime}(J) \frac{\partial X^{k}}{\partial x^{i}} \frac{\partial X^{l}}{\partial x^{j}} D_{k} D_{l} u^{j}
$$


or as

$$
J \frac{\partial X^{k}}{\partial x^{i}} D_{k} w^{\prime}(J)=J \partial_{i} w^{\prime}(J)=-\frac{P^{\prime}(\rho)}{\rho} \partial_{i} \rho .
$$

Combining (2.2-2.3) and (2.9-2.10) produces the system of wave equations

$$
\begin{aligned}
D_{t}^{2} u^{i}= & A_{k l}^{i j}\left(I+\frac{\partial u}{\partial X}\right) D_{k l}^{2} u^{j}+\lambda^{2}\left[\operatorname{det}\left(I+\frac{\partial u}{\partial X}\right)\right]^{2} w^{\prime \prime}\left(\operatorname{det}\left[I+\frac{\partial u}{\partial X}\right]\right) \\
& \cdot\left(\left[I+\frac{\partial u}{\partial X}\right]^{-1}\right)_{k i}\left(\left[I+\frac{\partial u}{\partial X}\right]^{-1}\right)_{l j} D_{k l}^{2} u^{j},
\end{aligned}
$$

with which are associated the initial conditions

$$
u(0, X, \lambda)=u^{0}(X, \lambda), \quad D_{t} u(0, X, \lambda)=v^{0}(X, \lambda) .
$$

System $(2.12-2.13)$ is the initial-value problem for which it is desired to prove the uniform existence and convergence results mentioned in the introduction. In order to be able to do this by applying theorems from [6 and 7], (2.10) must be converted to a first-order symmetric hyperbolic system. Because the term that $\lambda^{2}$ multiplies can be expressed most conveniently with Eulerian derivatives, we will also convert to Eulerian derivatives throughout. As a first step, using (2.1), (2.7), and (2.9-2.11), system (2.12) can be written as

$$
\begin{aligned}
& D_{t} u^{i}=v^{i} \\
& D_{t} v^{i}=A_{k l}^{i j}(I+G) D_{k}\left(D_{l} u^{j}\right)-\lambda^{2} \frac{P^{\prime}(\rho)}{\rho} \partial_{i} \rho, \\
& D_{t} \rho=-\rho \partial_{i} v^{i} .
\end{aligned}
$$

Next, taking $D_{k}$ of (2.14) and multiplying the result by $A_{k l}^{i j}$ gives

$$
A_{k l}^{i j} D_{t} G_{k}^{i}=A_{k l}^{i j} D_{k} v^{i}
$$

Defining $r \equiv \lambda \int_{1}^{\rho}\left(P^{\prime}(s) / s\right) d s$ and rewriting (2.19) and (2.16) in terms of $r$ gives,

$$
\begin{aligned}
& D_{t} v^{i}=A_{k l}^{i j} D_{k} G_{l}^{j}-\lambda \partial_{i} r, \\
& \frac{1}{P^{\prime}(\rho(r / \lambda))} D_{t} r=-\lambda \partial_{i} v^{i} .
\end{aligned}
$$

Converting the Lagrangian derivatives in (2.17-2.19) to Eulerian ones now gives

$$
\begin{gathered}
A_{k l}^{i j} \partial_{t} G_{k}^{i}+A_{k l}^{i j} v^{m} \partial_{m} G_{k}^{i}-\left(A_{k l}^{i j}\right)\left(\delta_{k}^{m}+G_{k}^{m}\right) \partial_{m} v^{i}=0, \\
\partial_{t} v^{i}+v^{m} \partial_{m} v^{i}-\left(A_{k l}^{i j}\right)\left(\delta_{k}^{m}+G_{k}^{m}\right) \partial_{m} G_{l}^{j}+\lambda \partial_{i} r=0, \\
\frac{1}{P^{\prime}(\rho(r / \lambda))} \partial_{t} r+\frac{1}{P^{\prime}(\rho(r / \lambda))} v^{m} \partial_{m} r+\lambda \partial_{i} v^{i}=0 .
\end{gathered}
$$

Note that although system $(2.20-2.22)$ is considered in a Eulerian coordinate 
system, its variables are Lagrangian derivatives of the original variables $u$. This system has the form

$$
A^{0}(V, V / \lambda) \partial_{t} V+A^{j}(V, V / \lambda) \partial_{j} V+\lambda B^{j} \partial_{j} V=0
$$

where $A^{0}$, the $A^{j}$, and the $B^{j}$ are symmetric matrices, and the $B^{j}$ are constant. Assumptions (1.4) and (1.6) will guarantee that $A^{0}$ is positive definite, so (2.23) is a symmetric hyperbolic system. The initial data for the unknown $V=(G, v, r)$ are

$$
\begin{aligned}
& G(0, x, \lambda)=G^{0}(x, \lambda)=\left.\frac{\partial u^{0}(X, \lambda)}{\partial X}\right|_{X=x(0, x, \lambda)}, \\
& v(0, x, \lambda)=v^{0}(x, \lambda)=\left.v^{0}(X, \lambda)\right|_{X=x(0, x, \lambda)}, \\
& r(0, x, \lambda)=r(x, \lambda)=\lambda \int_{1}^{1 / J^{0}(x, \lambda)} \frac{P^{\prime}(s)}{s} d s,
\end{aligned}
$$

which can be abbreviated as

$$
V(0, x, \lambda)=V^{0}(x, \lambda)
$$

\section{Uniform Existence and the Incompressible Limit}

Theorem 1. System (2.12-2.13) has, for $\lambda_{0} \leqq \lambda<\infty$, a solution $u$ in $C^{2}\left([0, T] \times R^{n}\right)$ for some $T$ independent of $\lambda$ provided that for some $m \geqq[n / 2]+3$,

i) $w \in C^{m+1}$ and there exists $W_{\infty} \in C^{m+1}$ such that $A_{k l}^{i j}(F)=\partial^{2} W_{\infty} / \partial F_{k}^{i} \partial F_{l}^{j}$;

ii) $w^{\prime \prime}(1)>0$ and $\left\{\operatorname{det}\left(I+\left(\partial u^{0} / \partial X\right)\right) \mid X \in R^{n}, \lambda \in\left[\lambda_{0}, \infty\right)\right\} \subset \subset\left\{s \mid w^{\prime \prime}(s)>0\right\}$;

iii) $A_{k l}^{i j}(F) Z^{i k} Z^{j l} \geqq c_{1}|Z|^{2}$ with $c_{1}>0$, for $F$ in a neighborhood $N$ of the identity matrix, and $\left\{I+\left(\partial u^{0} / \partial X\right) \mid x \in R^{n}, \lambda \in\left[\lambda_{0}, \infty\right)\right\} \subset \subset N$;

iv) $\left\|u^{0}(X, \lambda)\right\|_{m}+\left\|v^{0}(X, \lambda)\right\|_{m-1}+\lambda\left\|\operatorname{det}\left(I+\left(\partial u^{0} / \partial X\right)\right)-1\right\|_{m-1} \leqq c_{2}$, where $c_{2}$ is any constant and \|\|$_{k}$ is the usual Sobolev space norm of order $k$; and

v) the map $X \rightarrow x(0, X, \lambda) \equiv X+u^{0}(X, \lambda)$ is invertible for $\lambda_{0} \leqq \lambda<\infty$. Also, the function $\tilde{x}(X)=X+u(X)$ is an acceptable deformed position function, i.e. the map $X \rightarrow \tilde{x}$ is $1-1$. The solution $u$ satisfies the estimate

$$
\|u\|_{m}+\left\|D_{t} u\right\|_{m-1}+\frac{1}{\lambda}\left\|D_{t}^{2} u\right\|_{m-2}+\lambda\left\|\operatorname{det}\left(I+\frac{\partial u^{0}}{\partial X}\right)-1\right\|_{m-1} \leqq c,
$$

where here and later $c$ denotes a constant whose value may be different in different occurrences. Furthermore, if the initial data satisfies the additional condition

vi) $\lambda\left\|\left(\left[I+\frac{\partial u^{0}}{\partial X}\right]^{-1}\right)_{k i} D_{k}\left(v^{0}\right)^{i}\right\|_{m-2}+\lambda^{2}\left\|\operatorname{det}\left(I+\frac{\partial u^{0}}{\partial X}\right)-1\right\|_{m-1} \leqq c_{4}$,

where $c_{4}$ is any constant, then the solution satisfies the additional estimate

$$
\left\|D_{t}^{2} u\right\|_{m-2}+\lambda\left\|\left(\left[I+\frac{\partial u^{0}}{\partial X^{i}}\right]^{-1}\right)_{k i} D_{k} D_{t} u^{i}\right\|_{m-2}+\lambda^{2}\left\|\operatorname{det}\left(I+\frac{\partial u}{\partial X}\right)-1\right\|_{m-1} \leqq c .
$$


Remark. Let $J^{0}(X, \lambda)$ be the Jacobian determinant of the map in condition $\left.\mathrm{v}\right)$, and let $x \rightarrow \tilde{X}(0, x, \lambda)$ be the map's inverse. Condition vi) says that

$$
\lambda\left\|\partial_{i}\left[v^{0}(0, \tilde{X}(0, x, \lambda), \lambda)\right]^{i}\right\|_{m-2}+\lambda^{2}\left\|J^{0}(X, \lambda)-1\right\|_{m-1} \leqq C_{4} .
$$

This condition would be met, for example by initial data independent of $\lambda$ such that $\partial_{i}\left(v^{0}\right)^{i} \equiv 0$ and $J^{0} \equiv 1$, which, following [6], may be termed incompressible initial data.

Theorem 2. Assume that conditions i)-vi) of Theorem 1 hold and that in addition vii) there exist $u^{0}(X), v^{0}(X)$, such that

$$
\left\|u^{0}(X, \lambda)-u^{0}(X)\right\|_{m}+\left\|v^{0}(X, \lambda)-v^{0}(X)\right\|_{m-1} \rightarrow 0 \quad \text { as } \quad \lambda \rightarrow \infty .
$$

Then there exist $u^{\infty}$ in $C^{0}\left([0, T] ; H^{m}\right) \cap C^{1}\left([0, T] ; H^{m-1}\right) \cap C^{2}\left([0, T] ; H^{m-2}\right)$, and $r^{\infty}$ in $L^{\infty}\left([0, T] ; H^{m-1}\right)$, such that as $\lambda \rightarrow \infty$,

$$
\begin{aligned}
& u(t, X, \lambda) \rightarrow u^{\infty}(t, X) \text { in } C^{0}\left([0, T] ; H_{\mathrm{loc}}^{m-\varepsilon}\right) \cap C^{1}\left([0, T] ; H_{\mathrm{loc}}^{m-1-\varepsilon}\right), \\
& \lambda^{2}\left[\operatorname{det}\left(I+\frac{\partial u}{\partial x}\right)\right]^{2} w^{\prime \prime}\left(\operatorname{det}\left[I+\frac{\partial u}{\partial X}\right]\right)\left(\left[I+\frac{\partial u}{\partial X}\right]^{-1}\right)_{k i}\left(\left[I+\frac{\partial u}{\partial X}\right]^{-1}\right)_{l j} D_{k l}^{2} u^{j} \\
& \quad \rightarrow\left(\left[I+\frac{\partial u^{\infty}}{\partial X}\right]^{-1}\right)_{k i} D_{k} r^{\infty} \text { weak* in } L^{\infty}\left([0, T] ; H^{m-2}\right),
\end{aligned}
$$

for any $\varepsilon>0$. Also, $u^{\infty}$ and $r^{\infty}$ satisfy the initial-value problem of incompressible elastodynamics

$$
\begin{aligned}
D_{t}^{2}\left(u^{\infty}\right)^{i} & =A_{k l}^{i j}\left(I+\frac{\partial u^{\infty}}{\partial X}\right) D_{k l}^{2}\left(u^{\infty}\right)^{j}+\left(\left[I+\frac{\partial u^{\infty}}{\partial X}\right]^{-1}\right)_{k i} D_{k} r^{\infty}, \\
\operatorname{det}\left(I+\frac{\partial u^{\infty}}{\partial X}\right) & =1,
\end{aligned}
$$

with initial condition

$$
u^{\infty}(0, X)=u^{0}(X), \quad D_{t} u^{\infty}(0, X)=v^{0}(X) .
$$

Proof of Theorem 1. Assumption v) of Theorem 1 allows system (2.12-2.13) to be converted into system $(2.20-2.22,2.24-2.26)$, where $X(0, x, \lambda)$ in $(2.24-2.25)$ and $J^{0}(X, \lambda)$ in 2.26 are defined in the remark above. Apply the following theorem, slight variations of which appear in $[6,7$, and 8$]$ :

Theorem 3. System $(2.23,2.27)$ has, for $\lambda_{0} \leqq \lambda<\infty$, a unique solution in $C^{1}\left([0, T] \times R^{n}\right)$, where $T$ is independent of $\lambda$, provided that for some $q \geqq[n / 2]+2$

a) $A^{0}$, the $A^{j}$, and the $B^{j}$ are symmetric;

b) $A^{0}$ and the $A^{j}$ are in $C^{q}$ and the $B^{j}$ are constant matrices;

c) $A^{0} \geqq c_{1}>0$ in a neighborhood of the initial data,

i.e. $\left\{\left(V^{0}(x, \lambda), V^{0}(x, \lambda) / \lambda\right) \mid x \in R^{n}, \lambda \in\left[\lambda_{0} \infty\right)\right\} \subset \subset\left\{(a, b) \mid A^{0}(a, b) \geqq c_{1}>0\right\}$.

d) $\left\|V^{0}(x, \lambda)\right\|_{q} \leqq c_{2}$ for some $c_{2}$;

e) $\left[A^{0}(V, V / \lambda)\right]^{-1} B^{j}$ are functions of $V / \lambda$ only, not of $V$;

and either

f) $\left(\left.(\partial / \partial z) A^{0}(z, V / \lambda)\right|_{z=V}\right)\left(A^{0}\right)^{-1} B^{j}=0, \quad j=1, \ldots, n$; 
or

g) $\lambda\left\|B^{j} \partial_{j} V^{0}(x, \lambda)\right\|_{q-1} \leqq c_{3}$ for some $c_{3}$.

If a)-e) and f) hold, then the solution $V$ satisfies

$$
\|V\|_{q}+\frac{1}{\lambda}\left\|\partial_{t} V\right\|_{q-1} \leqq c
$$

while if a)-e) and g) hold, then

$$
\|V\|_{q}+\left\|\partial_{t} V\right\|_{q-1}+\lambda\left\|B^{j} \partial_{j} V\right\|_{q-1} \leqq c .
$$

Assumptions i)-iv) of Theorem 1 for system (2.12-2.13) imply conditions a) -d) of Theorem 3 for system $(2.20-2.22,2.24-2.26)$ with $q=m-1$. It is straightforward to check the key point that system (2.20-2.22) obeys the structural conditions e) and f) of Theorem 3. And if additional assumption vi) of Theorem 1 holds, then condition g) of Theorem 3 will hold.

Hence a solution $(G, v, r)$ of system $(2.20-2.22,2.24-2.26)$ exists on a uniform time interval. The proposed solution $u$ of $(2.12-2.13)$ is now obtained as the solution of the ODE, $D_{t} u(t, X, \lambda)=v(t, X+u, \lambda), u(0, X, \lambda)=u^{0}(X, \lambda)$. Since $v$ is bounded in $C^{0}$ uniformly in $\lambda$ by the Sobolev inequalities, $u$ exists for as long as $v$ is defined, i.e. on an interval $[0, T]$ independent of $\lambda$.

Define $\tilde{x}(t, X, \lambda)=X+u(t, X, \lambda)$, and define $\tilde{u}(t, x, \lambda)$ by $\partial_{t} \tilde{u}(t, x, \lambda)+$ $v^{k}(t, x, \lambda) \partial_{k} \tilde{u}(t, x, \lambda)=v(t, x, \lambda), \tilde{u}(0, x, \lambda)=u^{0}(X(0, x, \lambda), \lambda)$; this is a linear symmetric hyperbolic system, so $\tilde{u}$ is well-defined for $t$ in $[0, T]$. Define $\tilde{X}(t, x, \lambda)=x-\tilde{u}(t, x, \lambda)$. It is easy to check that $\tilde{X}(0, \tilde{x}(0, X, \lambda), \lambda)=X$ and $D_{t} \tilde{X}(t, \tilde{x}(t, X, \lambda) \lambda)=0$, so that $\tilde{X}$ is the inverse of $\tilde{x}$ for each $t$ and $\lambda$. In particular, $\tilde{x}(t, X, \lambda)$, which represents the position of time $t$ of the particle whose reference position is $X$, is $1-1$.

Next, for any function $f(t, x, \lambda), D_{t} f(t, \tilde{x}(t, X, \lambda), \lambda)=\partial_{t} f+v^{k} \partial_{k} f$, so that $(2.20$, 2.22) become the ODEs,

$$
\begin{aligned}
D_{t} G_{l}^{i}(t, \tilde{x}(t, X, \lambda), \lambda) & =\left(\delta_{l}^{m}+G_{l}^{m}\right) \partial_{m} v^{i}, \\
D_{t} r(t, \tilde{x}(t, X, \lambda), \lambda) & =-\lambda P^{\prime}(\rho(r / \lambda)) \partial_{i} v^{i},
\end{aligned}
$$

where $\partial_{k} v^{i}=\left.\partial_{k} v^{i}(t, x, \lambda)\right|_{x=\tilde{x}(t, X, \lambda)}$ is considered known.

Defining $\widetilde{G}_{l}^{i}=D_{l} v^{i}$ and $\tilde{r}=\lambda \int_{1}^{1 / \operatorname{det}(I+(\partial u / \partial X))}\left(P^{\prime}(s) / s\right) d s$, it is easy to show that $\widetilde{G}$ and $\tilde{r}$ satisfy the same equations as $G$ and $r$, respectively; since they also have the same initial conditions, $G=\widetilde{G}$ and $r=\tilde{r}$ by the uniqueness theorem for ODEs. Hence $D_{l}$, which equals $\left(\delta_{l}^{m}+\widetilde{G}_{l}^{m}\right) \partial_{m}$ by construction, also equals $\left(\delta_{l}^{m}+G_{l}^{m}\right) \partial_{m}$. Thus, converting (2.21) into Lagrangian coordinates and substituting $G=\tilde{G}, r=\tilde{r}$ produces $(2.12)$. Clearly (2.13) holds, so $u$ solves $(2.12-2.13)$ as claimed, and the estimates for $u$ follow from those for $(G, v, r)$.

Proof of Theorem 2. Convert to system (2.20-2.22), (2.24-2.26). The analogous result for this system is just a slight variation of Theorem 1 of [6] or Theorem 2.4 of [8]. Since there are no large terms in the equations for the additional unknowns $G$ that are present here, $G$ actually converges in $C^{1}$, and so the proof of convergence of $v$ is not affected by their presence. In order for the proof to show convergence as $\lambda \rightarrow \infty$ rather than convergence of a subsequence, uniqueness must hold for the limiting system; this uniqueness holds by a standard $L^{2}$ energy-estimate argument. The result 
is then pulled back to the original system exactly as for Theorem 1 .

Remarks on Theorem 3. The point of structural conditions e) and f) is that they prevent terms of size $O(\lambda)$ from occurring in energy estimates for the system, so that the energy estimates will be uniform in $\lambda$. Also, either of $\mathrm{f}$ ) or g) guarantees that $\partial_{t} A^{0}$ is $O(1)$ not $O(\lambda)$, so that $A^{0}$ remains positive definite on a uniform time interval. The proof when $g$ ) is assumed is given in [6 and 7]; the proof when $\mathrm{f}$ ) is assumed is given in [7 and 8]. Note in this regard that although [7 and 8] have instead of e) and f) the stronger hypotheses $A^{0}=A^{0}(v / \lambda)$, the proof they give requires only assumptions e) and $\mathrm{f}$ ). This trivial strengthening of the theorem is important because it is only with the hypotheses used here that the theorem applies to the elasticity equations $(2.20-$ 2.22).

Acknowledgements. I thank Profs. Sergiu Klainerman and Robert V. Kohn for their generous assistance, which included both suggesting this problem and providing me with a copy of their unpublished work on it.

\section{References}

1. Ball, J. M.: Remarks on the Paper "Basic calculus of variations." Pac. J. Math. (to appear)

2. Ebin, D.: Motion of slightly compressible fluids in a bounded domain I. Commun. Pure Appl. Math. 35, 451-485 (1982)

3. Gurtin, M. E.: An introduction to continuum mechanics. New York: Academic Press 1981

4. Hughes, T. J. K., Kato, T., Marsden, J. E.: Well-posed quasi-linear hyperbolic systems with applications to nonlinear elastodynamics and general relativity. Arch. Ration. Mech. Anal. 63, 273294 (1977)

5. John, F.: Finite amplitude waves in a homogeneous isotropic elastic solid. Commun. Pure Appl. Math. 30, 421-446 (1977)

6 Klainerman, S., Majda, A.: Singular limits of quasilinear hyperbolic systems with large parameters and the incompressible limit of compressible fluids. Commun. Pure Appl. Math. 34, 481-524 (1981)

7. —- Compressible and incompressible fluids, Commun. Pure Appl. Math. 35, 637-656 (1982)

8. Majda, A.: Compressible fluid flow and systems of conservation laws in several space dimensions. Berlin, Heidelberg, New York: Springer 1984

9. Marcellini, P.: Quasiconvex quadratic forms in two dimensions. Appl. Math. Options (to appear)

10. Rostamian, R. Internal constraints in boundary value problems of continuum mechanics, Indiana Univ. Math. J. 27, 637-656 (1978)

11. Schochet, S.: The compressible Euler equations in a bounded domain: existence of solutions and the incompressible limit. (to appear)

12. Terpstra, F. J.: Die darstellong biquadratischer formen als summen von quadraten mit unwendung aut die variations rechaung. Math. Ann. 116, 166-180 (1938)

13. Truesdell, C., Noll, W.: The nonlinear field theories of mechanics. In: Handbook der Physik III/I, Flogge, S. (ed.) Berlin, Heidelberg, New York: Springer 1965

14. Uhlig, F.: A recurring theorem about pairs of quadratic forms and extensions: a survey. Linear Algebra Appl. 25, 219-237 (1979)

15. Edin, D.: The motion of slightly compressible fluids viewed as a motion with strong constraining force. Ann. Math. 105, 141-200 (1977)

Communicated by L. Nirenberg

Received December 27, 1984; in revised form June 10, 1985 
